\title{
$\beta$-Mannosidase Deficiency: Heterogeneous Manifestation in the First Female Patient and her Brother
}

\author{
W. J. Kleijer ${ }^{1}$, P. Hu ${ }^{1}$, R. Thoomes ${ }^{1}$, M. Boer ${ }^{1}$, J. G. M. Hujumans ${ }^{2}$, \\ W. Blom ${ }^{2}$, O. P. VAN Diggelen ${ }^{1}$, E. Seemanova ${ }^{3}$ and M. MaceK ${ }^{3}$ \\ ${ }^{1}$ Department of Clinical Genetics, University Hospital, Erasmus University, \\ PO Box 1738, 3000 DR Rotterdam, The Netherlands; ${ }^{2}$ Department of Paediatrics, \\ Sophia Children's Hospital, Erasmus University, Rotterdam, The Netherlands; \\ ${ }^{3}$ Research Institute of Child Development, Department of Medical Genetics, \\ Charles University, Prague, Czechoslovakia
}

\begin{abstract}
Summary: $\beta$-Mannosidase deficiency was demonstrated in fibroblasts of a girl who showed severe psychomotor retardation, bone deformities and gargoylism and recurrent skin and respiratory infections and who died at 20 years of age from bronchopneumonia. This first demonstration of a female patient confirms the autosomal recessive inheritance of $\beta$-mannosidosis. Further investigation of this gipsy family revealed $\beta$-mannosidosis in an older brother with a milder manifestation of gargoyl facial dysmorphology, mental retardation, hearing impairment and recurrent infections. $\beta$-Mannosidase activity was completely deficient in his cultured skin fibroblasts, leukocytes and plasma. In urine a characteristic disaccharide was present. Heterozygote levels of $\beta$-mannosidase were found in fibroblasts and/or plasma of the parents and one sister.
\end{abstract}

$\beta$-Mannosidosis (McKusick 24851) is a lysosomal storage disease caused by the deficiency of $\beta$-mannosidase (EC 3.2.1.25). The disorder was first described in goats as a severe and rapidly fatal neurological disease (Jones and Dawson, 1981; Lovell and Jones, 1983). The human counterpart was reported simultaneously by Cooper and colleagues (1986) in two adult brothers and by Wenger and colleagues (1986) in a 4-year-old boy. Subsequently, Dorland and colleagues (1988) described two brothers of 7 and 9 years of age with elevated urinary levels of the disaccharide mannosyl$\beta(1 \rightarrow 4)$ - $\mathrm{N}$-acetylglucosamine and $\beta$-mannosidase deficiency.

The patients described thus far are all mentally retarded but further manifestations are heterogeneous and much milder than in the caprine disease. Since the report of $\beta$-mannosidosis in goats, several groups have searched for the human disease by screening patients with neurological and presumably lysosomal disease. We did not detect this enzyme deficiency in a series of 40 stored cell lines from young patients

*Correspondence

MS received 21.3.90 Accepted 16.5.90 
with progressive neurological deterioration (Panday et al., 1984). More recently we studied another series of cell lines from older patients or those with generally milder symptoms and demonstrated $\beta$-mannosidosis in a girl who was 13 years of age at the time of skin biopsy. This first female patient and her older affected brother are reported in this paper.

\section{PATIENTS AND METHODS}

Clinical: The proband (II-8; Figure 1) was one of dizygous twins in a sibship of nine children. The gipsy parents originate from two small neighbouring villages in Czechoslovakia but consanguinity was not proven. One child (II-3) died from respiratory disease at six months of age. All the other children are mentally retarded to various degrees. In three of them the retardation is mild and no other abnormalities are reported (II-4,5 and 9). Five children have grown up in institutions for oligophrenic children (II-1,2,6,7 and 8), one of whom could not be traced (II-1). Clinical data for the other four children are summarized below.

II-8: The female proband was born in 1966 and died in 1986 from bronchopneumonia and sepsis. Psychomotor retardation was apparent from early life. The girl could crawl only at 14 months of age, could sit only at 18 months, was very hypotonic, and was severely mentally retarded. From the fourth year onwards there are reports of recurrent skin and respiratory infections, severe anaemia, epistaxis and automutilation such as the tearing of hairs leading to local alopecia. Further features were gargoyl facies, hypertelorism, macroglossia, gingival hyperplasia, short neck, umbilical hernia and skeletal abnormalities (deformities of the thorax, lumbar hyperlordosis and nanism: height $1.10 \mathrm{~m}$ at 18 years of age; weight $22 \mathrm{~kg}$ ) and increased fragility of capillaries. There was no hepatosplenomegaly.

The EEG showed a lack of basal activity. Haematological examination showed slight vacuolization and granulation of bone marrow cells. X-ray examination showed broad ribs, pelvic asymmetry, spina bifida occulta and severe scoliosis.

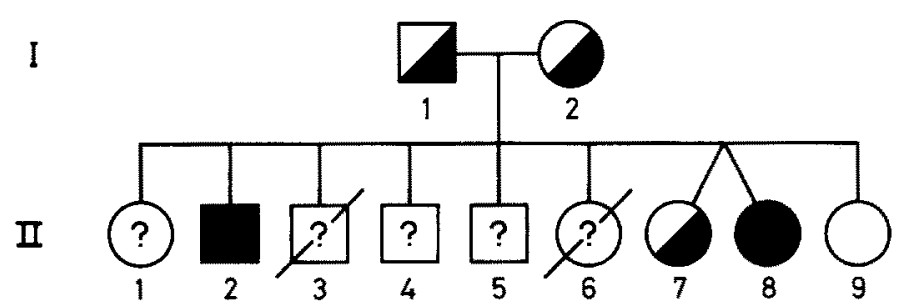

Figure 1 Pedigree of the family with $\beta$-mannosidosis. $\beta$-Mannosidase activity is deficient in II- 2 and 8 , reduced in the parents and II-7 and normal in II-9. Siblings II-1,3,4,5 and 6 were not available for study. 
II-2: This older brother is moderately mentally retarded and shows occasional aggressive behaviour. Hearing is impaired; facial features are coarse with slight hypertelorism and thick lips but are not so clearly gargoyl-like as in proband II-8. Slight scoliosis was observed by $\mathrm{X}$-ray examination. There are frequently recurring erysipelas-like skin changes and respiratory infections. His height is normal: $1.83 \mathrm{~m}$ at 30 years of age.

II-6: This sister was born in 1964 and died in 1980 with severe dystrophy and terminal bronchopneumonia. She had microcephaly with low frontal port of the skull and was severely mentally retarded; she could sit only at six years of age. Muscular hypotony and scoliosis were reported as well as coarse facies with a broad mouth, but not gargoyl-like as in proband II-8.

II-7: This twin sister is also severely mentally retarded with dyslalia due to perinatal encephalopathy. From her fourth year she suffered from paroxysmal epilepsy. There is no facial dysmorphology as in proband II-8.

Biochemical studies: Fibroblasts were cultured from a skin biopsy of proband II- 8 in 1979 and have been stored in the cell bank. No other material was available for recent studies as the patient died in 1986. Blood and/or skin biopsies have been collected from the parents (I-1 and 2) and from the siblings II-2,7 and 9. Urine was collected from sibling II-2. No material could be obtained from the other siblings, because they either could not be traced (II-1,4 and 5) or had died (II-3,6).

$\beta$-Mannosidase activity was assayed as described previously (Panday et al., 1984). Other lysosomal enzyme activities and $\left[{ }^{35} \mathrm{~S}\right]$-sulphate incorporation levels were measured according to the method of Galjaard (1980). Thin layer chromatography of oligosaccharides was performed on silica coated plates using solvent 1 as described by Blom and colleagues (1983) (n-butanol-acetic acid-water, 2:1:1 by volume) or solvent 2 as described by Dorland and colleagues (1988) (n-propanol-acetic acidwater, $85: 1: 15$ by volume). The TLC plate was developed twice in the same solvent.

\section{RESULTS}

Enzyme studies: The clinical features of proband II-8 suggested a lysosomal storage disease, perhaps mucolipidosis type II or III. Skin fibroblasts were cultured and investigated for several lysosomal enzyme activities. Normal activities were established for $\beta$-galactosidase, $\alpha$-mannosidase, $\alpha$-fucosidase, $\alpha$-glucosidase and $\alpha$-N-acetylneuraminidase (values not shown), which excluded mucolipidosis II and III and the isolated enzyme deficiencies as well. As a general test for most known types of mucopolysaccharidoses, $\left[{ }^{35} \mathrm{~S}\right]$-sulphate incorporation in glycosaminoglycans was determined and found to be normal. Finally, $\beta$-mannosidase activity in the patient's fibroblasts was shown to be completely deficient (Table 1 ). By the time this $\beta$ mannosidase deficiency was found, the patient had died and urine, blood or tissues were not available for further studies. Three of the patient's siblings and her parents could be traced and were prepared to donate a skin biopsy, blood or urine. 
Table $1 \beta$-Mannosidase activity in cultured fibroblasts, leukocytes and plasma

\begin{tabular}{lccc}
\hline & Fibroblasts $^{\mathrm{a}}$ & Leukocytes $^{\mathrm{a}}$ & Plasma $^{\mathrm{b}}$ \\
\hline Patient, II-8 & 0.0 & - & - \\
Patient, II-2 & 0.6 & 0.9 & 0.1 \\
Sister, II-7 & 29 & 59 & 54 \\
Sister, II-9 & - & - & 90 \\
Father, I-1 & 45 & - & 79 \\
Mother, I-2 & 28 & - & 67 \\
Controls, mean \pm SD & $91 \pm 30$ & $85 \pm 15$ & $138 \pm 20$ \\
$\quad$ Range & $51-195$ & $45-150$ & $87-188$ \\
$n$ & 76 & 42 & 48 \\
\hline
\end{tabular}

${ }^{a} \mathrm{nmol} / \mathrm{h}$ per mg protein; ${ }^{b} \mathrm{nmol} / \mathrm{h}$ per ml; ${ }^{\circ}$ The pedigree is shown in Figure 1

$\beta$-Mannosidase appeared to be deficient in fibroblasts, leukocytes and plasma from the brother (II-2), whereas reference lysosomal enzymes were normal. Fibroblasts and plasma of the twin sister (II-7) and the parents had a reduced enzyme activity suggesting heterozygosity. Of the youngest sister (II-9) only plasma could be stndied; $\beta$-mannosidase was normal (Table 1).

Heparan sulphate sulphamidase activity was normal in fibroblasts of both patients with $\beta$-mannosidase deficiency (data not shown).

Urine analysis: Urine from patient II-2, sister II-9 and their parents was analysed for oligosaccharides. Figure 2 shows an abnormal band in the oligosaccharide pattern on TLC of the urine of patient II-2. The abnormal oligosaccharide coincides with lactose if solvent 1 is used, whereas it is separated from lactose in solvent 2 . The patterns for sister II-9 and the parents were normal.

\section{DISCUSSION}

$\beta$-Mannosidase deficiency in two siblings from a large gipsy family, described in this paper, was associated with mental retardation, hearing impairment, recurrent infections of the skin and respiratory tract and gargoyl-like facial dysmorphism. The female proband (II-8, Figure 1) was severely affected in these and several other respects which became fatal at 20 years of age, whereas the brother is not so severely affected.

It is difficult to identify a consistent pattern of clinical manifestations among the seven patients in the four families described until now, including the present cases. Various degrees of mental retardation and hearing loss with associated impairment of speech are common findings. Aggressive behaviour was mentioned in one of the adult patients described by Cooper and colleagues (1990) and in our adult patient (II-2). Isolated observations are angiokeratoma in the brothers described by Cooper and colleagues $(1986,1988)$. Our two patients had recurrent infections of the skin (erysipelas) and respiratory tract; respiratory infections were also observed in the patients described by Dorland and colleagues (1988). Coarse facies and skeletal abnormalities were present in our two patients and in the patient described by 


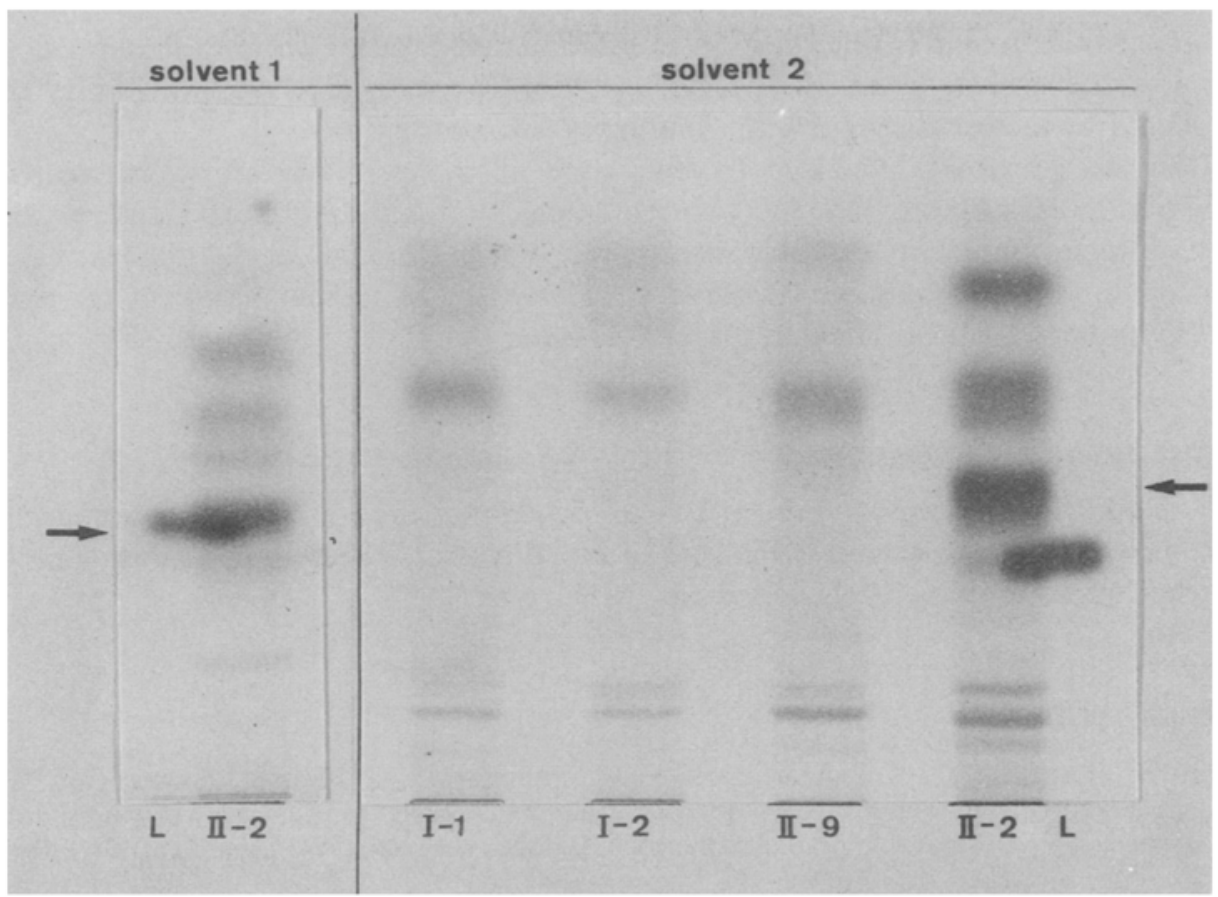

Figure 2 Thin layer chromatography of urinary oligosaccharides from $\beta$-mannosidosis patient II-2 and his healthy sister II-9 and parents I-1 and 2. The extra band in the patient (see arrow) coincides with the lactose standard (L) in solvent 1 but is separated from lactose in solvent 2.

Wenger and colleagues (1986). However, in the latter patient at least, some of the clinical manifestations may be attributed to a second disorder, Sanflippo disease type A, which was indicated by a complete deficiency of heparan sulphate sulphamidase. This enzyme deficiency was not present in any of the other patients including the two described here.

In the present family the severe psychomotor retardation, hypotonia and bone anomalies in the proband contrast remarkably with the milder presentation of these features in her affected brother and the other reported $\beta$-mannosidosis patients. At least one sister (II-6, Figure 1) died with complications as severe as in the proband, but the possible involvement of $\beta$-mannosidosis could not be investigated. The causal pathogenesis of the signs and symptoms of disease in this family is further complicated by mental retardation due to perinatal encephalopathy in the proband's non-identical twin sister (II-7), in whom $\beta$-mannosidosis was excluded by a heterozygote level of enzyme activity.

In spite of active searching by several groups, only four families with $\beta$-mannosidosis have been found since the discovery of the caprine disease (Jones and Dawson, 1981). This suggests that the disorder is quite rare. However, due to the severe neurological manifestation of the disease in goats, the disorder may initially have been searched for mainly among patients with very early onset of severe neurological complications. Also, the specific trisaccharide demonstrated in the urine of affected goats is not 
present in the human counterpart. Instead, in human patients variable amounts of a urinary disaccharide occur (Dorland et al., 1988; Cooper et al., 1990), which may be obscured by lactose during routine thin layer chromatography.

The five previously reported patients were all males. Nevertheless, autosomal inheritance is suggested by the reduced levels of $\beta$-mannosidase activity in the heterozygous parents (e.g. this paper, Table 1 and Dorland et al., 1988) and by consanguinity in one couple (Dorland et al., 1988). The present report of the first female patient confirms autosomal recessive inheritance.

\section{ACKNOWLEDGEMENTS}

We thank Mrs E. van Mol and Mr E. v.d. Meijden for their help in oligosaccharide analysis and Dr F. Kothe and the staff of the 'Bloedbank Rotterdam' for providing blood samples from control individuals.

\section{REFERENCES}

Blom, W., Luteijn, J. C., Kelholt-Dijkman, H. H., Huijmans, J. G. M. and Loonen, M. C. B. Thin layer chromatography of oligosaccharides in urine as a rapid indication for the diagnosis of lysosomal acid maltase deficiency (Pompe's disease). Clin. Chim. Acta 134 (1983) $221-227$

Cooper, A., Sardharwalla, I. B. and Roberts, M. M. Human $\beta$-mannosidase deficiency. $N$. Engl. J. Med. 315 (1986) 1231

Cooper, A., Hatton, C., Thornley, M. and Sardharwalla, I. B. Human $\beta$-mannosidase deficiency: Biochemical findings in plasma, fibroblasts, white cells and urine. J. Inher. Metab. Dis. 11 (1988) 17-29

Cooper, A., Hatton, C. E., Thornley, M. and Sardharwalla, I. B. $\alpha$ - and $\beta$-Mannosidoses. $J$. Inher. Metab. Dis. 13 (1990) 538-548

Dorland, L., Duran, M., Hoefnagels, F. E. T., Breg, J. N., Fabery de Jonge, H., Cransberg, K., van Sprang, F. J. and van Diggelen, O. P. $\beta$-Mannosidosis in two brothers with hearing loss. J. Inher. Metab. Dis. 11 Suppl. 2 (1988) 255-258

Galjaard, H. Genetic Metabolic Disease: Early Diagnosis and Prenatal Analysis, Elsevier, Amsterdam and New York, 1980

Jones, M.Z. and Dawson, G. Caprine $\beta$-mannosidosis: Inherited deficiency of $\beta$-D-mannosidase. J. Biol. Chem. 256 (1981) 5185-5188

Lovell, K. L. and Jones, M. Z. Distribution of central nervous system lesions in $\beta$-mannosidosis. Acta Neuropathol. 62 (1983) 121-126

Panday, R. S., van Diggelen, O. P., Kleijer, W. J. and Niermeijer, M. F. $\beta$-Mannosidase in human leucocytes and fibroblasts. J. Inher. Metab. Dis. 7 (1984). 155-156

Wenger, D. A., Sujansky, E., Fennessey, P. V. and Thompson, J. N. Human $\beta$-mannosidase deficiency. N. Engl. J. Med. 315 (1986) 1201-1205 